\title{
Rambach agar and SM-ID medium sensitivity for presumptive identification of Salmonella subspecies I-VI
}

\author{
SARINA PIGNATO, GIOVANNI GIAMMANCO* and GIUSEPPE GIAMMANCO†
}

Istituto d'Igiene e Medicina Preventiva, Università di Catania, Via Biblioteca 4, 95124 Catania and * Dipartimento d'Igiene e Microbiologia "G. D'Alessandro", Sezione di Microbiologia. Università di Palermo, Via Del Vespro 133, 90127 Palermo, Italy

\begin{abstract}
Summary. The cultural characteristics of 112 Salmonella serovars belonging to subspecies I-VI were examined on Rambach agar and SM-ID medium. Colonies showing the typical red coloration were seen with 100 of 112 serovars assayed on SM-ID, and with 87 of 112 on Rambach agar. Atypical colourless colonies were observed on Rambach agar with ONPGnegative serovars $S$. Choleraesuis, $S$. Isangi, $S$. Typhi $S$. Worthington and $S$. Yoff of the subspecies I. S. II $52: \mathrm{d}: \mathrm{e}, \mathrm{n}, \mathrm{x}, \mathrm{z}_{15}$ of the subspecies II, $S$. IV $6,7: \mathrm{z}_{1}, \mathrm{z}_{24}:-$ and $S$. IV $11: \mathrm{g}, \mathrm{z}_{51}:-$ of the subspecies IV, and $S .40: z_{35}$ :- belonging to $S$. bongori $(\mathrm{V})$ species. Atypical blue, bluegreen, blue-violet or violet colonies were observed on both media with all the ONPG-positive serovars of the subspecies IIIa (four of four strains) and IIIb (six of six strains) and with one of the two ONPG-positive (out of five) strains of the subspecies VI. Four serovars of $S$. bonyori showed blue-green colonies on Rambach agar and typical red colonies on SM-ID, although they were all ONPG-positive. These results suggest that SM-ID medium is more sensitive than Rambach agar. However, the relatively low sensitivity of both media makes them suitable for use only in association with a traditional selective medium in both medical and environmental bacteriology, as well as for epidemiological purposes.
\end{abstract}

\section{Introduction}

Rambach agar (E. Merck) and SM-ID medium (bioMérieux) are new selective media for the isolation of salmonellae.

Rambach agar ${ }^{1}$ uses the ability of Salmonella species to produce acid from propylene glycol to differentiate them from other enteric bacteria. In addition, the presence of the chromogenic substrate X-Gal (5-bromo-4-chloro-3-indolyl- $\beta$-D-galactopyranoside) allows the detection of $\beta$-D-galactosidase production by other members of the Enterobacteriaceae. Salmonella spp. cultures are positive only in the first of these reactions and grow as bright red colonies on Rambach agar, with the exception of $S$. Typhi, which is negative in tests for propylene glycol acidification and shows colourless colonies. Escherichia coli and Klebsiella spp. are positive only for $\beta$-D-galactosidase production and appear as blue colonies. Proteus spp. give negative results in both reactions, so the colonies are colourless. Citrobacter freundii appears violet as a result of the combination of colours from the two reactions.

Received I Sept. 1994: revised version accepted 14 Jan. 1995 +Correspondence should be sent to Professor G. Giammanco.
The biochemical characteristic used in SM-ID medium $^{2}$ is acid formation from glucuronate in addition to $\beta$-D-galactosidase production. Salmonella spp. colonies have a red colouration on this medium, while Citrobacter spp. colonies become blue because of the precipitation of a chromogenic substrate by $\beta$-Dgalactosidase. E. coli gives positive results in both reactions and so appears violet whereas Proteus spp. perform neither reaction and so are colourless.

Some Salmonella serovars are reported not to form colonies of the expected colour on Rambach agar, ${ }^{3-5}$ possibly as a result of the presence of $\beta$-D-galactosidase activity. Since this biochemical characteristic should be restricted to some of the subspecies of Salmonella, the ONPG reaction of a number of collection strains that represented the full range of species and subspecies were tested and the results were compared to the colour of the colonies on both Rambach agar and SM-ID medium, in order to compare the sensitivity of these two media for the detection of salmonellae.

\section{Materials and methods}

One hundred and twelve different serovars of Salmonella were tested: 74 of them belonged to 
subspecies 1, including $S$. Typhi, 12 to subspecies II, four to subspecies IIIa, six to subspecies IIIb, five to subspecies IV, five to subspecies VI and six to the species $S$. bongori $(\mathrm{V})$. All strains were typed by conventional serological methods. The ONPG test was performed by a conventional method. A number of strains came from the collection of the Unite des Entérobactéries, Institut Pasteur, Paris (France); the others had been isolated from stool and food samples in the laboratories of the Istituto d'Igiene e Medicina Preventiva of the University of Catania (Italy).

Broth cultures were prepared and, after incubation for $6 \mathrm{~h}$ at $37^{\circ} \mathrm{C}$, a loopful was streaked on each of the two media under examination. The colour of the streaks and colonies were observed after incubation for $24 \mathrm{~h}$ at $37^{\circ} \mathrm{C}$.

\section{Results}

The characteristics of inoculation streaks and isolated colonies of the different Salmonella strains assayed on Rambach agar and SM-ID medium are reported in the table, together with the results of the ONPG test.

Of 74 serovars of subspecies I, the following 69 showed typical cultures on both media: Agona, Anatum, Anatum var. 15+, Arechavaleta, Bellevue, Berta, Bispebjerg, Blockley, Bovismorbificans, Braenderup, Brancaster, Brandenburg, Bredeney, Cerro, Chester, Coeln, Corvallis, Derby, Dublin, Emek, Enteritidis, Essen, Gallinarum, Glostrup, Goldcoast, Haardt, Hadar, Haifa, Heidelberg, Idikan, Indiana, Infantis, Javiana, Kapemba, Kinshasa, Lichfield, Livingstone, Lomita, London, Mara, Meleagridis, Montevideo, Muenchen, Muenster, Napoli, Nashua, Newport, Niarembe, Obogu, Ohio, Onderstepoort, Panama, Paratyphi A (two strains), Paratyphi B (three strains), Pensacola, Reading, Rutgers, Saintpaul, Sandiego, Schleissheim, Senftenberg, Telaviv, Thompson, Typhimurium, Typhimurium var. Copenhagen, Virchow, Virginia, Wien, Yaba.

Streaks and isolated colonies of red colour were obtained on SM-ID medium with ONPG-negative serovars belonging to the subspecies I, II and IV, with the sole exception of two serovars of subspecies IV whose streaks developed an orange colour. More exceptions were observed on Rambach agar, on which five subspecies I serovars (Choleraesuis, Isangi, Typhi, Worthington and Yoff) and one subspecies II serovar (II 52:d:e,n, $x, z_{15}$ ) showed colourless cultures, whereas the other serovars developed a typical red colour. Furthermore, the streaks of the five subspecies IV strains and the isolated colonies of two of these strains were colourless on this medium. The ONPG-positive strains of the subspecies IIIa and IIIb showed blue, blue-green, blue-violet, violet or mauve streaks and colonies on Rambach agar and a violet or blue-violet colour on SM-ID. Different results were observed on the two media with the $S$. bongori (V) strains, which showed a blue-green colour on Rambach agar and a red colour on SM-ID medium, although they were all ONPG-positive, with the exception of two strains that produced blue-violet colonies on SM-ID medium and colourless colonies on Rambach agar, respectively. Three ONPG-negative serovars of the subspecies VI showed red colonies on Rambach agar and SM-ID, as did one of the two ONPG-positive serovars, while the other produced blue-green and blue-violet colonies on the same media.

\section{Discussion}

Traditional selective media for the isolation of salmonellae, such as SS agar, desoxycholate-citrate agar and Hektoen enteric agar, rely on lactose fermentation and hydrogen sulphide production to differentiate salmonellae from non-pathogenic enteric bacteria. However, the specificity of these media is low because the biochemical properties utilised are common to other enterobacterial species such as Proteus spp. and lactose-negative Citrobacter freundii, which frequently inhabit the intestinal tract. The identifying characteristics of Rambach agar are acid production from propylene glycol (a phenotypic property specific for Salmonella spp.) and $\beta$-D-galactosidase production by other members of the Enterobacteriaceae. Unfortunately, some important Salmonella serovars such as $S$. Typhi ${ }^{1}$ are unable to form acid from propylene glycol, while some subspecies possess $\beta$-Dgalactosidase and develop colonies indistinguishable from coliform cultures. ${ }^{5}$ In this study, not only $S$. Typhi but also other serovars of the Salmonella subspecies I, II and IV produced colourless colonies and thus were indistinguishable. Furthermore, almost all the strains belonging to the ONPG-positive serovars of the subspecies IIIa, IIIb and VI as well as to $S$. bongori (V) species produced blue cultures that were indistinguishable from coliform bacteria. In contrast, all the serovars assayed in the subspecies I, II, IV, including $S$. Typhi, and in $S$. bongori (V) (with only one exception) showed typical red colonies when cultured on SM-ID. Therefore, this medium appears to be more sensitive than Rambach agar. Nevertheless, ONPG-positive strains of subspecies IIIa and IIIb were indistinguishable from coliform bacteria with both Rambach agar and SM-ID.

The great majority of the serovars responsible for human infections belongs to the subspecies I. ${ }^{6.7}$ Therefore, Rambach and SM-ID media could both be recommended for practical purposes on the grounds of their high specificity. ${ }^{8}$ However, their relatively low sensitivity and inability to detect $S$. Typhi on Rambach agar, and the majority of the ONPGpositive serovars on both media, can cause difficulty. Further problems can occur if these media are used for the primary isolation of salmonellae from clinical specimens or food samples where the presence of other species in mixed culture could affect the colonial characteristics of the salmonellae. In particular, the 
Table. Characteristics of cultures of different Salmonella spp., subspecies and serovars on Rambach agar and SM-ID medium

\begin{tabular}{|c|c|c|c|c|c|}
\hline \multirow{2}{*}{$\begin{array}{l}\text { Species, subspecies } \\
\text { and serovars }\end{array}$} & \multirow{2}{*}{$\begin{array}{l}\text { ONPG } \\
\text { test }\end{array}$} & \multicolumn{2}{|c|}{ Colour on Rambach agar } & \multicolumn{2}{|c|}{ Colour on SM-ID medium } \\
\hline & & Streak & Single colonies & Streak & Single colonies \\
\hline \multicolumn{6}{|l|}{$\begin{array}{l}\text { S. enterica } \\
\text { subsp. enterica (I) }\end{array}$} \\
\hline N. 69 serovars (see text) & - & Red & Red & Red & Red \\
\hline Choleraesuis & - & Colourless & Colourless & Red & Red \\
\hline Isangi & - & Colourless & Colourless & Red & Red \\
\hline Typhi & - & Colourless & Colourless & Red & Red \\
\hline Worthington & - & Colourless & Colourless & Red & Red \\
\hline Yoff & - & Colourless & Colourless & Red & Red \\
\hline \multicolumn{6}{|l|}{ subsp. salamae (II) } \\
\hline II. $1.9 .12: 1 . w:$ e.n.x & - & Red & Red. light edge & Red & Red \\
\hline Westpark & - & Red & Red & Red & Red \\
\hline II $4,12: b:-$ & - & Red & Red, light edge & Red & Red \\
\hline Tosamanga & - & Red & Red & Red & Red \\
\hline II, $6.7: \mathrm{z}_{29} ;-$ & - & Colourless & Red & Red & Red \\
\hline II $9.12: 1$, v:e.n,x & - & Red & Red & Red & Red \\
\hline Haarlem & - & Red & Red & Red & Red \\
\hline Haddon & - & Red & Red & Red & Red \\
\hline II $35: \mathrm{d}: 1.5$ & - & Red & Red & Red & Red \\
\hline II $42: z_{10}: 1.2$ & - & Red & Red & Red & Red \\
\hline Il $52: \mathrm{d}: \mathrm{e}, \mathrm{n}, \mathrm{x}, \mathrm{z}_{15}$ & - & Colourless & Colourless & Red & Red \\
\hline Basel & - & Colourless & Red, light edge & Red & Red \\
\hline \multicolumn{6}{|l|}{ subsp. arizonae (IIIa) } \\
\hline IIIa $42: g: z_{j 1}$ & + & Blue-green & Blue-green & Blue & Blue \\
\hline IIIa $42: z_{4}, z_{24}:-$ & + & Violet & Blue-violet & Violet & Violet, red edge \\
\hline IIIa $56: z_{29}:-$ & + & Blue-violet & Blue-violet & Violet & Violet \\
\hline IIIa $59: z_{4}, z_{23}:-$ & + & Mauve & Mauve & Violet & Violet, red edge \\
\hline \multicolumn{6}{|l|}{ subsp. diarizonae (IIIb) } \\
\hline IIIb $11: 1, v: z_{\bar{i} 3}$ & + & Mauve & Blue-violet & Blue-violet & Blue-violet \\
\hline IIIb $14: 1 . v: z$ & + & Blue & Blue-violet & Blue-violet & Blue-violet \\
\hline IIIb $16: 1 . v: z_{533}$ & + & Blue & Blue-violet & Blue-violet & Blue-violet \\
\hline IIIb $48: \mathrm{k}: 1,5^{3.3}$ & + & Blue & Blue & Blue-violet & Blue-violet \\
\hline IIIb $57: \mathrm{c}: z$ & + & Mauve & Mauve & Blue-violet & Blue-violet \\
\hline \multicolumn{6}{|l|}{ subsp. houtenae (IV) } \\
\hline IV $6.7: z_{4}, z_{24}:-$ & - & Colourless & Colourless & Red & Red \\
\hline IV $11: \mathrm{g} . z_{51}:-$ & - & Colourless & Colourless & Orange & Red \\
\hline IV $16: z_{4}, z_{23}:-$ & - & Colourless & Red, light edge & Red & Red \\
\hline IV $48: z_{1}, z_{23}:-$ & - & Colourless & Red, light edge & Orange & Red \\
\hline IV $51: \mathrm{b}:-$ & - & Colourless & Red, light edge & Red & Red \\
\hline \multicolumn{6}{|l|}{ subsp. indica (VI) } \\
\hline VI 1 i:a: 1.5 & - & Red & Red & Red & Red \\
\hline V1 $1,6,14,25: a: e, n, x$ & + & Red-crimson & Red, light edge & Blue-green & Red \\
\hline VI $1,6,14.25: z_{10}: 1,(2) .7$ & - & Red & Red & Orange & Red \\
\hline VI $41: b: 1.7$ & + & Blue-green & Blue-green & Blue-violet & Blue-violet \\
\hline VI $45: a: e, n, x$ & - & Colourless & Red & Orange & Red \\
\hline \multicolumn{6}{|l|}{ S. bongori (V) } \\
\hline $\mathrm{V} 40: z_{35}:-$ & + & Colourless & Colourless & Red & Red \\
\hline V $48: z_{35}:-$ & + & Blue-green & Blue-green & Red & Red \\
\hline Camdeni & + & Blue-green & Blue-green & Red & Red \\
\hline Balboa & + & Blue-green & Blue-green & Red & Red \\
\hline Maregrosso & + & Blue-green & Blue-green & Red & Red \\
\hline Brookfield & + & Blue-green & Blue-green & Blue-violet & Blue-violet \\
\hline
\end{tabular}

red colonial coloration could be lost through rapid realkalinisation of the media as a result of the strong glucolytic activity of some strains ${ }^{8}$ or profuse growth of proteolytic organisms. It is therefore inadvisable to rely on these media alone for the detection of Salmonella spp. in either diagnostic medical bacteriology or in epidemiological and environmental in- vestigations. The use of SM-ID or Rambach agar in parallel with a traditional selective medium would seem to be the most appropriate procedure on the basis of these results.

This work was supported by a grant (Finanziamento Ricerca Scientifica $40 \%$ ) from the Italian Ministry for the University and Technological Research.

\section{References}

1. Rambach A. New plate medium for facilitated differentiation of Salmonella spp. from Proteus spp. and other enteric bacteria. Appl Environ Microbiol 1990; 56: 301-303.

2. Poupart MC. Mounier M, Denis F, Sirot J, Couturier C.
Villeval F. A new chromogenic ready-to-use medium for Salmonella detection. 5th European Congress of Clinical Microbiology and Infectious Diseases, Oslo. Norway, 1991.

3. Gruenewald R, Henderson RW, Yappow S. Use of Rambach propylene glycol containing agar for identification of Salmonella spp. J Clin Microbiol 1991; 29: 2354-2356. 
4. Freydiere AM, Gille Y. Detection of salmonellae by using Rambach agar and by a C8 esterase spot test. $J$ Clin Microbiol 1991; 29: 2357-2359.

5. Kühne H, Wonde B, Rabsch W, Reissbrodt R. Evaluation of Rambach agar for detection of Salmonella subspecies I to VI. Appl Environ Microbiol 1994; 60: 749-751.

6. Le Minor L, Grimont PAD. Origine et répartition en sérovars des souches de Salmonella isolées en France continental au cours des années 1984 à 1987. Médicine et Maladies Infectieuses $1989 ; 19: 12-17$.

7. Center for Disease Control. Salmonella surveillance--annual summary. Centers for Disease Control, Atlanta, 1989.

8. Dusch H, Altwegg M. Comparison of Rambach agar, SM-ID medium, and Hektoen enteric agar for primary isolation of non-typhi salmonellae from stool samples. J Clin Microbiol $1993 ; 31: 410-412$. 